International Journal of Engineering \& Technology, 7 (2.21) (2018) 50-52
International Journal of Engineering \& Technology
SPC
Website: www.sciencepubco.com/index.php/IJET
Research paper

\title{
Hybrid synchronisation of vallis chaotic systems using nonlinear active control
}

\author{
Piyush Pratap Singh *, Vikash Kumar, Eshan Tiwari, Vinay K. Chauhan \\ Dept. of Electrical Engineering, NIT Meghalaya, Shillong, Meghalaya, India. \\ *Corresponding author E-mail: piyushpratapsingh@gmail.com
}

\begin{abstract}
In this paper, hybrid synchronisation of Vallis chaotic systems using a nonlinear control technique is proposed. Vallis system represents the principal quantitative features of the El-Nino Southern Oscillation (ENSO) phenomenon. A nonlinear active control technique is used for hybrid synchronisation. Control laws are designed by using the sum of the relevant variables of the both mater and slave systems. Required Lyapunov stability condition is devised using Lyapunov stability theory. Numerical simulation results reflect the successful achievement of the proposed objectives. MATLAB is used for simulation.
\end{abstract}

Index Terms: Chaotic System, ENSO, hybrid synchronisation, lyapunov stability Theory, vallis System.

\section{Introduction}

Sensitivity to initial conditions is a generic feature of chaotic dynamical systems. Two chaotic systems starting from slightly different initial points in the state space separate away from each other with time. Therefore, controlling of two chaotic systems to make them synchronised has aroused a great deal of interest. The idea of synchronizing two identical chaotic systems starting from different initial conditions was introduced by Pecora and Carroll [1]. Synchronisation of chaotic systems has been extensively investigated in the last few years and many possible applications have been discussed using computer simulation and realised in laboratory condition.

The researchers widely use drive-response synchronisation. In this scheme, the output of drive system is used to control the response system. Synchronisation means to achieve zero error between states of drive and response systems. Another important and similar phenomenon between two dynamical systems is antisynchronisation. During anti-synchronisation state of both drive and response systems show the same amplitude but opposite in phase. Anti-synchronisation causes the sum of state of drive and response systems converge asymptotically to zero. Similarly, the hybrid synchronisation phenomenon means coexistence of synchronisation and anti-synchronisation both, i.e., some of the states are anti-synchronised and the rest are synchronised.

In 2008, C. Li et al. [2] investigated the coexistence of antisynchronisation along with synchronisation, i.e. some of the state variables were synchronised and the rest anti-synchronised. In this paper pair of states of coupled Chen system are anti-phase synchronised, and the rest is complete synchronised by nonlinear active linear control via a single variable. Sufficient conditions for the hybrid synchronisation were obtained in terms of asymptotic stability. The work by Sudheer et al. [3, 4] investigated the hybrid synchronisation of hyperchaotic Chen systems [3] and hyperchaotic Lu systems [4] in 2009. Nonlinear active control is designed to achieve hybrid synchronisation between drive and response systems using the sum and difference of relevant variables of the chaotic systems. In 2012, Sundarapandian et al. $[5,6]$ presented hybrid synchronisation of hyperchaotic Lorenz systems and hyperchaotic Chen systems [5] in 2011 and chaotic Arneodo and Rossler systems [6], etc.

G. K. Vallis [7] investigated the Vallis system which represents the principal quantitative features of the ENSO phenomenon. These phenomena are the occurrence of sea-surface warmings in the eastern equatorial pacific and the associated trade wind reversal. It is the three variables conceptual model, namely near surface temperatures in the east and west equatorial ocean and a wind driven current advecting the temperature field. For a large range of parameters, the model is chaotic and aperiodically produces El-Nino like events. El-Nino is the occurrence of an anomalously warm pool of the water in the eastern equatorial Pacific Ocean. In 1988, G. K. Vallis [8] further considered the lower order continuous models, with and without external stochastic forcing and reveals that even in the absence of stochastic forcing, chaos and aperiodic ENSO events can occur. After a long time, in 2014, a maiden attempt is made by $\mathrm{J}$. Samantaray et al. [9] to investigate the anti-synchronisation of Vallis system [7] when $\mathrm{p}=0$. In the year 2015 , the work by B. M. Garay et al. [10] the Vallis' symmetric and asymmetric Lorenz models [8] for El-Nino systems of autonomous ordinary differential equations in 3D with the usual parameters are considered. In this paper, they located topological horseshoes in iterates of Poincare return maps based on the standard Mischaikow-Mrozek-Zgliczynski approach.

Literature reveals that the flow of the water motion on the surface of ocean, temperatures in western and eastern parts of the ocean need synchronisation and anti-synchronisation using appropriate control technique. Also, it is apparent from the paper [9] that the fluctuations of the temperature in western and eastern parts of near equatorial area of the ocean are the major concern and may be further improved significantly. Therefore, motivated with these issue, an attempt is made to achieve hybrid synchronisation of Vallis chaotic systems, in this paper.

Rest of the paper is organised as follows. A brief description is presented about Vallis system in Section 2. In the Section 3, 
hybrid synchronisation using a nonlinear active control is demonstrated and stability analysis is achieved. Results and discussion is given in the Section 4. Finally, Section 5 reveals the conclusions and future scope.

\section{Description of vallis chaotic system}

Vallis chaotic system (talks about eastern and western temperature fluctuation and flow of water motion) are the major part of research. The Vallis system is given by the following equation.

$\left\{\begin{array}{l}\dot{x}=-a x+\mathrm{u} y \\ \dot{y}=-y+x z \\ \dot{z}=1-x y-z\end{array}\right.$

where $x$ is the water motion on a surface of ocean and

$$
\left\{\begin{array}{l}
y=\left[T_{w}-T_{e}\right] / 2 \\
z=\left[T_{w}+T_{e}\right] / 2 .
\end{array}\right.
$$

$T_{w}$ and $T_{e}$ are temperatures in western and eastern parts of ocean, respectively. $\mathrm{u}$ and $a$ are positive parameters of the Vallis system (1). The phase plane behaviour of Vallis system is shown in the Fig. (1) which reflects the chaotic behaviour.
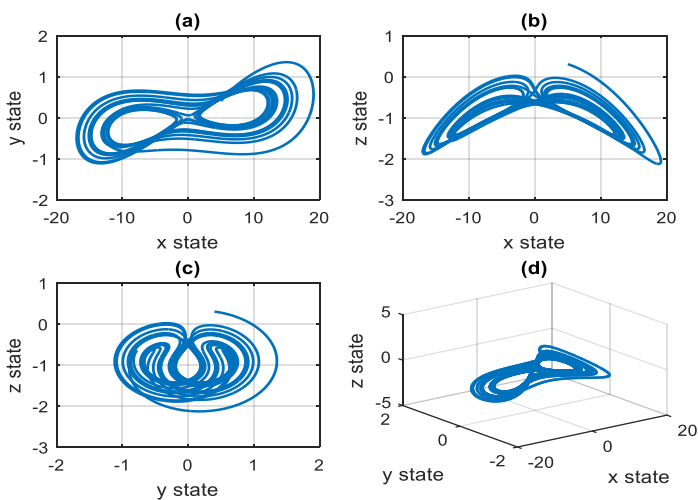

Fig. 1: Chaotic attractors of the vallis system

\section{Hybrid synchronisation of vallis chaotic Systems}

Vallis chaotic system is considered as master as well as slave systems. The master and slave systems are defined in (2) and (3), respectively, as:

$\left\{\begin{array}{c}\dot{x}_{m 1}(t)=-a x_{m 1}(t)+u x_{m 2}(t) \\ \dot{x}_{m 2}(t)=x_{m 1}(t)-x_{m 2}(t)+x_{m 1} x_{m 3}(t) \\ \dot{x}_{m 3}(t)=-x_{m 1}(t) x_{m 2}(t)-x_{m 3}(t)\end{array}\right.$

and

$\int \dot{x}_{s 1}(t)=-a x_{s 1}(t)+u x_{s 2}(t)+u_{1}(t)$

$\left\{\dot{x}_{s 2}(t)=x_{s 1}(t)-x_{s 2}(t)+x_{s 1}(t) x_{s 3}(t)+u_{2}(t)\right.$

$\dot{x}_{s 3}(t)=-x_{s 1}(t) x_{m 2}(t)-x_{s 3}(t)+u_{3}(t)$

where, $\boldsymbol{u}_{1}, \boldsymbol{u}_{2}$ and $\boldsymbol{u}_{3}$ are the control laws added to slave system.

To achieve hybrid synchronisation, the error is defined as given in (4).

$\left\{\begin{array}{l}e_{1}(t)=x_{m 1}(t)+x_{s 1}(t) \\ e_{2}(t)=x_{m 2}(t)-x_{s 2}(t) \\ e_{3}(t)=x_{m 3}(t)+x_{s 3}(t)\end{array}\right.$

It may be noted that the first and third states are antisynchronising and the rest is synchronising. The error dynamics is written as:

$\left\{\begin{array}{l}\dot{e}_{1}(t)=\dot{x}_{m 1}(t)+\dot{x}_{s 1}(t) \\ \dot{e}_{2}(t)=\dot{x}_{m 2}(t)-\dot{x}_{s 2}(t) \\ \dot{e}_{3}(t)=\dot{x}_{m 3}(t)+\dot{x}_{s 3}(t)\end{array}\right.$
Further, the above error dynamics is modified as:

$$
\left\{\begin{array}{c}
\dot{e}_{1}(t)=-a_{1}(t)+u\left[x_{m 2}(t)+x_{s 2}(t)\right]+u_{1}(t) \\
\dot{e}_{2}(t)=-e_{2}(t)+x_{m 1}(t)-x_{s 1}(t)\left[1+x_{s 3}(t)\right]+x_{m 1}(t) x_{m 3}(t)-u_{2}(t) \\
\dot{e}_{3}(t)=-x_{s 1}(t) x_{s 2}(t)+x_{m 1}(t) x_{m 2}(t)-e_{3}(t)+u_{3}(t)
\end{array}\right.
$$

The objective is to design a nonlinear active control to guarantee the error, $\boldsymbol{e}_{\boldsymbol{i}}(\boldsymbol{t})$ between the states of the model (master) system and plant (slave) system tends to zero asymptotically, as time tends to infinite.

\section{Stability analysis of vallis chaotic system while hybrid synchronisation}

Let the suitable control laws $\boldsymbol{u}_{\mathbf{1}}(\boldsymbol{t}), \boldsymbol{u}_{\mathbf{2}}(\boldsymbol{t}), \boldsymbol{u}_{\mathbf{3}}(\boldsymbol{t})$ be defined as:

$\left\{\begin{array}{c}u_{1}(t)=-u\left[x_{m 2}(t)+x_{s 2}(t)\right] \\ u_{2}(t)=x_{m 1}(t) x_{m 3}(t)-x_{s 1}(t) x_{s 3}(t)+e_{1}(t) \\ u_{3}(t)=x_{s 1}(t) x_{s 2}(t)+x_{m 1}(t) x_{m 2}(t)\end{array}\right.$

Using the control inputs $\boldsymbol{u}_{1}(\boldsymbol{t}), \boldsymbol{u}_{2}(\boldsymbol{t})$ and $\boldsymbol{u}_{3}(\boldsymbol{t})$, the modified error dynamics is written as:

$\left\{\begin{array}{l}\dot{e}_{1}(t)=-a e_{1}(t) \\ \dot{e}_{2}(t)=-e_{2}(t) \\ \dot{e}_{3}(t)=-e_{3}(t)\end{array}\right.$

Let a Lyapunov function candidate be defined as:

$V(e)=\frac{1}{2}\left(e_{1}^{2}+e_{2}^{2}+e_{3}^{2}\right)$

Assuming if $\boldsymbol{V}(\boldsymbol{e})$ is continuously differentiable function, then we have

$\dot{V}(e)=e_{1}(t) \dot{e}_{1}(t)+e_{2}(t) \dot{e}_{2}(t)+e_{3}(t) \dot{e}_{3}(t)$

As putting the value in above function, we get $\dot{\boldsymbol{V}}(\boldsymbol{e})$ as:

$\dot{V}(e)=-a e_{1}^{2}(t)-e_{2}^{2}(t)-e_{3}^{2}(t)$

$\dot{\boldsymbol{V}}(\boldsymbol{e})<\boldsymbol{0}$ i.e. the origin is asymptotically stable. In addition, $\boldsymbol{V}(\boldsymbol{e})$ is radially unbounded. Therefore, the origin is globally asymptotically stable [11] while hybrid synchronisation.

\section{Results and discussions}

MATLAB is used for numerical simulation. Initial condition for master and slave systems are considered as $\left[\begin{array}{lll}4 & 0.4 & 0.3\end{array}\right]^{T}$ and $\left[\begin{array}{lll}1 & 2 & 1\end{array}\right]^{T}$, respectively. Simulation is done for 10 seconds with step size 0.005 . The time and frequency domain analyses are achieved and shown in the Fig. 2.

The required control inputs and synchronisation errors are depicted in the Figs. 3(a) and 3(b), respectively. Time domain response in the Fig. 2 reveals that the hybrid synchronisation is achieved successfully. Synchronisation errors shown in the Fig. 3(b) reflects that the objective is achieved using the appropriate nonlinear active control inputs Fig. 3(a). Finally, the phase plane behaviours of the master and slave systems are shown in the Fig. 4 to have a simultaneous look while hybrid synchronisation.

Frequency domain analysis reveals the information about the parameters like leakage factor, sidelobe attenuation and mainlobe width for each of the states of the master and slave systems and summarised in the Table 1.

\section{Conclusions and future scope}

In this paper, hybrid synchronisation between the Vallis chaotic systems using nonlinear active control technique is achieved. 
Nonlinear active control laws are designed successfully to achieve hybrid synchronisation. The designed control laws insure the stability of the error dynamics. Globally asymptotically stability condition is derived by using Lyapunov stability condition. Simulation is achieved in MATLAB and MATLAB results correspond the successful achievement of the proposed objectives. Therefore, it is concluded that the flow of the water motion on the surface of ocean, temperatures in western and eastern parts of the ocean are synchronisation and anti-synchronisation using appropriate nonlinear active control technique.Further improvement may be possible while hybrid synchronisation using other robust control design as a future work.

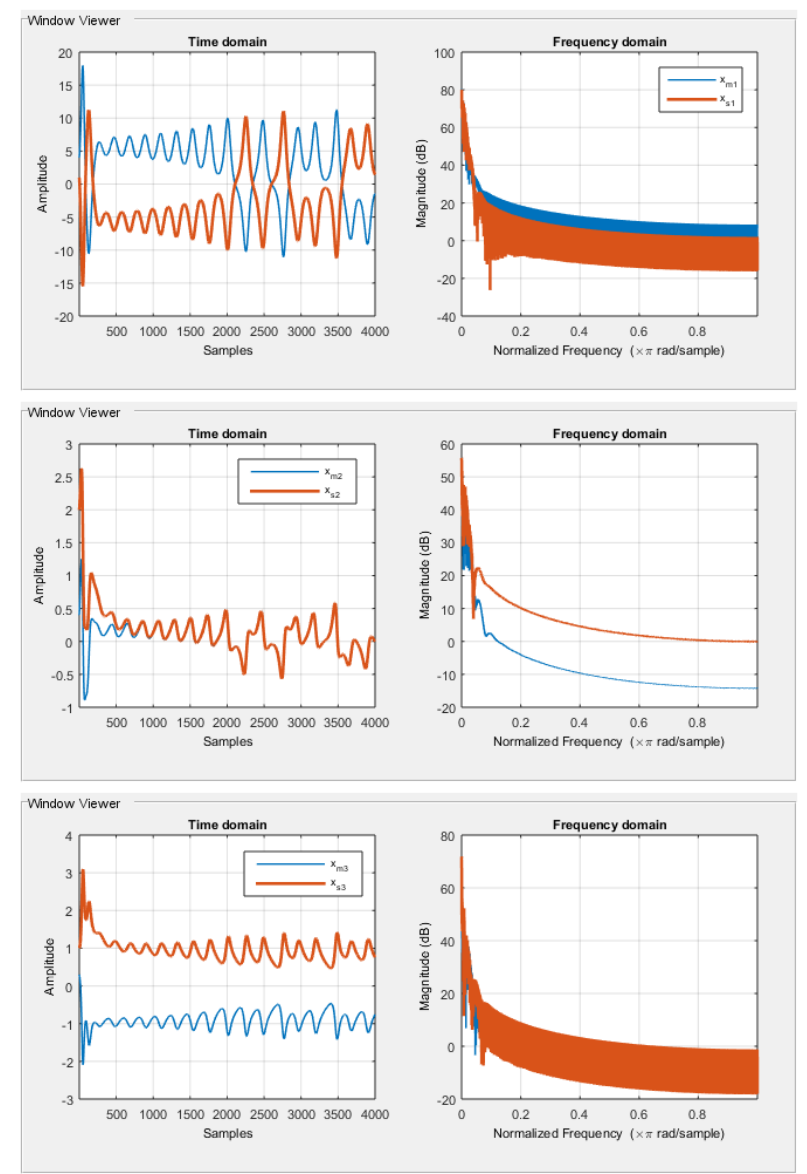

Fig. 2: Shows the time and frequency response behaviours of the master and slave systems

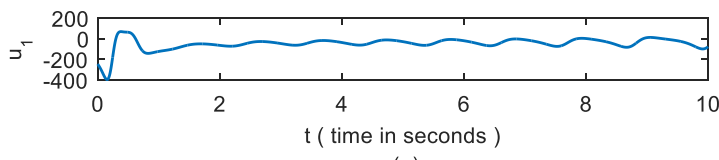

(a)

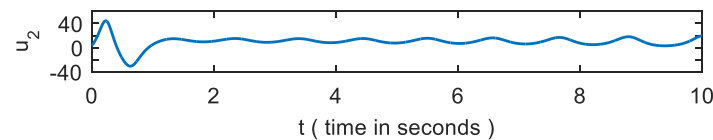

(b)

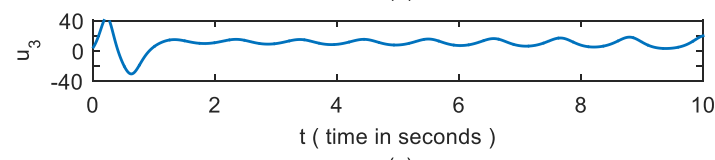

(c)

(a)

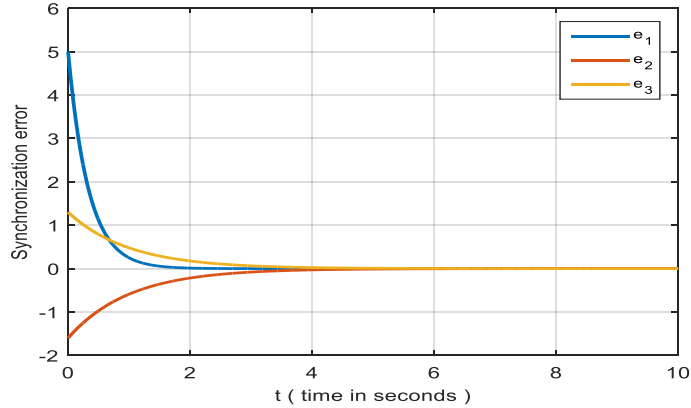

(b)

Fig. 3: Represents the control inputs in (a) and hybrid synchronisation errors in (b), between the mater and slave systems
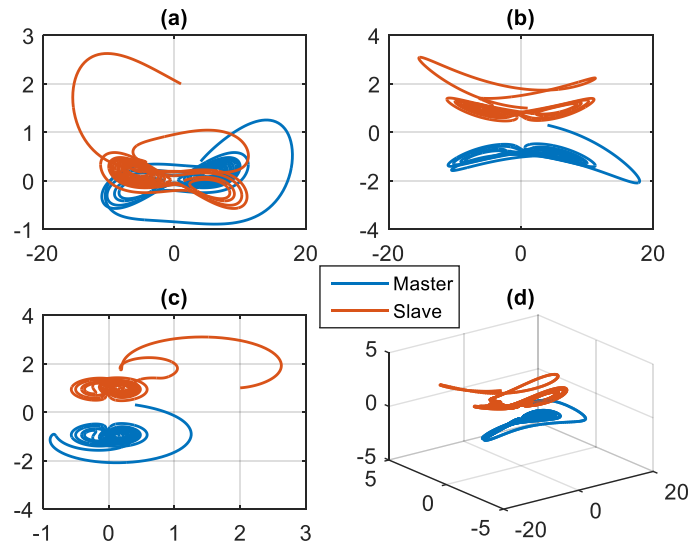

Fig. 4: Shows the phase behaviour of the master and slave vallis chaotic systems under hybrid synchronisation

Table 1: Frequency Domain Analysis

\begin{tabular}{|l|l|l|l|}
\hline Parameters & \multicolumn{4}{|l|}{ States of Master and Slave Systems } \\
\hline & First State & Second State & Third State \\
\hline Leakage factor $(\%)$ & 66.04 & 73.53 & 21.79 \\
\hline Sidelobe attenuation (dB) & -5.70 & 4.0 & -11.60 \\
\hline Mainlobe width (-3dB) & 0.00079346 & 0.00079346 & 0.00036621 \\
\hline
\end{tabular}

\section{References}

[1] Pecora LM \& Carrol TL, "Synchronisation of chaotic systems", Chaos: An Interdisciplinary Journal of Nonlinear Science, Vol.25, No.9, (2015).

[2] Li C, Chen Q \& Huang T, "Coexistence of anti-phase and complete synchronisation in coupled Chen system via single variable", Chaos, Solitons and Fractals, Vol.38, (2008), pp.461464.

[3] Sudheer KS \& Sabir M, "Hybrid synchronisation of hyperchaotic Chen system", National Conference on Nonlinear Systems Dynamics, Saha Institute of Nuclear Physics, (2009).

[4] Sudheer KS \& Sabir M, "Hybrid synchronisation of hyperchaotic Lu system", Pramana J. Phys., Vol.73, No.4, (2011), pp.781786.

[5] Sundarapandian V \& Karthikeyan R, "Hybrid chaos synchronisation of hyperchaotic Lorenz and hyperchaotic Chen systems by active nonlinear control", Int. J. Electr. Power Eng., Vol.5, No.5, (2011), pp.186-192.

[6] Sundarapandian V \& Rasappan S, "Hybrid synchronisation of Arneodo and Rossler chaotic systems by active nonlinear control", LNICST, Vol.84, No.1, (2012), pp.73-82..

[7] Vallis GK, "El Nino: a chaotic dynamical system?", Science, Vol. 232, (1986), pp.243-245.

[8] Valli GK, "Conceptual models of El Nino and the Southern Oscillation”, J Geophys Res Oceans, Vol.93, No.11, (1988).

[9] Samantaray J, Singh PP \& Roy BK, "Anti-synchronisation of Vallis system using Sliding Mode Control", J. Control Instrumentation, Vol.5, No.3, (2014), pp.22-27.

[10] Garay BM \& Indig B, "Chaos in Vallis' asymmetric Lorenz model for El Nino”, Chaos, Solitons \& Fractals, Vol.75, (2015), pp.253-262.

[11] Slotine JE \& Li W, Applied Nonlinear Control, New Jersey: Prentice Hall, (1991). 